\title{
La ingesta de aceite esencial de orégano (Lippia origanoides) mejora la morfología intestinal en Broilers
}

\author{
Madrid-Garcés, T.A. @; López-Herrera, A. y Parra-Suescún, J.E.
}

Facultad de Ciencias Agrarias, Universidad Nacional de Colombia, sede Medellín.

Palabras ClaVe adicionales

Broilers.

Lippia origanoides.

Criptas.

Vellosidades.

\section{RESUMEN}

Los antibióticos han sido utilizados como aditivos en los alimentos para animales, especialmente en pollos de engorde a nivel mundial, con fines terapéuticos y como promotores de crecimiento (APC);sin embargo, la creciente preocupación por la proliferación de microorganismos resistentes, ha llevado a la prohibición del uso de antibióticos en alimentación animal en muchos países. Como una alternativa, se ha propuesto el uso de extractos derivados de plantas aromáticas, denominados "aceites esenciales". Por lo anterior, el objetivo de este trabajo fue evaluar el efecto de la inclusión de tres niveles de Aceite Esencial de Orégano (AEO), (Lippia origanoides), sobre el crecimiento y la morfología digestiva en pollos de engorde de la línea genética Cobb500. Se utilizaron 200 pollos machos de un día de nacidos, alojados en corrales en piso. Los animales fueron aleatorizados a una de cinco dietas: dieta comercial con y sin antibiótico; esta última adicionada con diferentes niveles de AEO (0ppm, 75ppm, 100ppm o 200ppm AEO). Las aves fueron sacrificadas los días 14, 28 y 42 de vida, y se les extrajo el intestino delgado, del cual se tomaron porciones de duodeno, yeyuno e íleon para evaluación histológica. Se realizó un diseño estadístico de bloques al azar en un arreglo de parcelas dividas, donde los animales fueron aleatorizados a uno de 15 tratamientos (5 dietas por 3 edades). Para las vellosidades intestinales se encontró un aumento significativo en la longitud y ancho, y para las criptas intestinales una disminución significativa en la profundidad y ancho; ambos parámetros a favor de la dieta con mayor inclusión de AEO (200ppm AEO) $(P<0.01)$.La adición de 200ppm de AEO en el alimento de pollos de engorde aumenta la longitud y ancho de las vellosidades, y disminuye la profundidad y ancho de las criptas a nivel intestinal, aumentando la capacidad de absorción del intestino de las aves. Por lo anterior, el AEO se constituye como un promotor nutricional de crecimiento de origen vegetal, afectando positivamente la morfología intestinal durante el ciclo de producción de carne con pollos de engorde.

\section{Inclusion of essential oil of oregano enhances intestinal morphology in broiler}

\section{SUMMARY}

\section{ADDITIONAL KEYWORDS}

Broilers.

Lippia origanoides.

Crypts.

Villus.

\section{INFORMATION}

Cronología del artículo.

Recibido/Received: 05.11.2016

Aceptado/Accepted: 16.09.2018

On-line: 15.10 .2018

Correspondencia a los autores/Contact e-mail:

tamadridg@unal.edu.co
Antibiotics have been used as additives in animal feed, especially in broilers worldwide, for therapeutic purposes and as growth promoters (APC); However, the growing concern over the proliferation of resistant microorganisms has led to the prohibition of the use of antibiotics in animal feed in many countries. As an alternative, the use of extracts derived from aromatic plants, called "essential oils" has been proposed. Therefore, the objective of this work was to evaluate the effect of the inclusion of three levels of Oregano Essential Oil (AEO), (Lippia origanoides), on the growth and digestive morphology in broilers of the Cobb500 genetic line. We used 200 male chicks oneday old, housed in pens on the floor. The animals were randomized to one of five diets: commercial diet with and without antibiotic; this last one added with different levels of AEO (0ppm, 75ppm, 100ppm or 200ppm AEO). The birds were sacrificed on days 14,28 and 42 of life, and the small intestine was extracted, from which portions of the duodenum, jejunum and ileum were taken for histological evaluation. A randomized block statistical design was performed in an array of divided plots, where the animals were randomized to one of 15 treatments (5 diets for 3 ages). For intestinal villi a significant increase in length and width was found, and for intestinal crypts a significant decrease in depth and width was found; both parameters in favor of the diet with greater inclusion of AEO (200ppm AEO) ( $P<0.01)$. The addition of 200ppm of AEO in the feed of broilers increases the length and width of the villi, and decreases the depth and width of the crypts at the intestinal level, increasing the absorption capacity of the intestine of the birds. Therefore, the AEO is constituted as a nutritional growth promoter of plant origin, positively affecting the intestinal morphology during the meat production cycle with broiler chickens.

\section{INTRODUCCIÓN}

La alta demanda de una fuente de proteína segura y libre de agentes infecciosos está en ascenso en todo el mundo,sin embargo, durante el crecimiento intensivo, la industria avícola siempre se ha enfrentado a retos de diversas enfermedades. Bajo estas circunstancias, las mayores pérdidas económicas se deben a enfermedades infecciosas que podrían ser causadas por virus, bacterias, hongos o protozoos, y por ende, al costo 
de la medicación preventiva (Maguiña-Vargas, 2013). Esto dio lugar a un mayor uso de antibióticos en la industria de las aves de corral con fines terapéuticos, profilácticos y como promotores de crecimiento (APC) que pueden dejar residuos en los productos obtenidos de las aves de corral. La presencia de residuos de APC en la carne de aves de corral y los huevos puede tener efectos nocivos sobre los consumidores humanos (Martínez, Ruiz, \& R, 2008)

Durante las últimas décadas se utilizó una cantidad considerable de antibióticos en la producción animal, como agentes terapéuticos y promotores del crecimiento (Oloruntola, Ayodele, Agbede, \& Oloruntola, 2016.) lo que ha desencadenado preocupaciones sobre la resistencia a los antibióticos y la prohibición de los mismos en dosis sub-terapéuticas en la industria de aves de corral en Europa, y la posibilidad de una prohibición en los Estados Unidos, ha incrementado un gran interés en la búsqueda de alternativas naturales a los APC para la producción de aves de corral; dentro de estas alternativas se destacan los estractos naturales o fitobióticos (García-Curbelo \& García-Hernández, 2015). Cada vez es más importante encontrar nuevas formas de mantener la competitividad dentro de la industria mientras se genera un producto de alta calidad para los consumidores (Ojediran, Fasola, Oladele, Onipede, \& Emiola, 2017) lo que hace imperativo la utilización de productos que reemplacen los APC obteniendo los mismo o mejores resultados a nivel productivo.

El intestino tiene una superficie extensa, por lo que el animal orienta gran parte de los nutrientes consumidos y de la inmunidad hacia este órgano, ya que es la puerta de entrada de la mayoría de los patógenos al organismo del hospedero (Tavernari, Salguero, Albino, \& Rostagno, 2008). En comparación con los mamíferos, el tubo intestinal aviar es relativamente más pequeño con relación al peso corporal, ya que ha tenido que adaptarse para el vuelo. Esto está compensado con una mayor irrigación sanguínea, una secreción gástrica más alta, un tránsito digestivo más rápido y una acidez mayor. Además, el intestino de las aves tiene una mayor densidad de microvellosidades intestinales y un ritmo de reciclado epitelial más rápido que el de los mamíferos (Sergeant, y otros, 2014).(Cervantes 2011).

La altura y ancho de las vellosidades, además de la profundidad y ancho de las criptas en el intestino, pueden ser influenciadas por el alimento e ingredientes en la dieta. Esta influencia es más evidente en los primeros once días de vida, donde el ave desarrolla y capacita el intestino para su función digestiva. Las aves que poseen vellosidades más grandes (largas y anchas) tienen una mejor absorción de los nutrientes ya que generan mayor superficie de absorción (Skoufos, y otros, 2016).

El aceite esencial de orégano (AEO), de diferentes variedades, ha sido estudiado para la suplementación de pollos de engorde y hay reportes como el de Betancourt (2012) donde se camparan tres quimiotipos diferentes del orégano, concluyendo que los aceites esenciales de L. origanoides se constituyen en una alternativa económicamente viable para el desarrollo y producción de un aditivo natural que mejore condiciones de la producción de pollos de engorde y que reemplace de forma efectiva a los APC.

Por lo anterior, el objetivo de este trabajo fue evaluar el efecto de la inclusión a tres niveles de AEO de Lippia origanoides sobre el crecimiento y la morfología digestiva en pollos de engorde de la línea genética Cobb500.

\section{MATERIALES Y MÉTODOS}

\section{CONSIDERACIONES ÉTICAS}

Todos los procedimientos experimentales se llevaron a cabo de acuerdo a las guías propuestas por The International Guiding Principles for Biomedical ResearchInvolving Animals (CIOMS, 2012). Esta investigación fue avalada por el Comité de Ética en la Experimentación Animal de la Universidad Nacional de Colombia, Sede Medellín (CEMED 045 del 10 de junio de 2014).

\section{LOCALIZACIÓN}

El trabajo de campo se realizó en el Centro de producción San Pablo, perteneciente a la Universidad Nacional de Colombia, Sede Medellín, ubicado en el municipio de Rionegro, paraje "El Tablacito", localizado a $2100 \mathrm{msnm}$, con una temperatura entre 12 y $18^{\circ} \mathrm{C}$, correspondiendo a una zona de vida bosque muy húmedo Montano bajo (bmh-MB).

\section{ANIMALES}

Se utilizaron 200 pollos macho de línea Avian Cobb500 de un día de nacidos, alojados en corrales en piso. El período experimental tuvo una duración de 42 días. La cría se realizó siguiendo procedimientos comerciales en una granja experimental.

\section{MANEJo SANITARIO}

Para el recibimiento de los pollos, se realizó lavado, limpieza y desinfección del galpón, cortinas, comederos y bebederos; además, se hizo el control de roedores e insectos con productos obtenidos en casas comerciales. Las criadoras se encendieron cinco horas antes de la llegada de los animales.

El plan vacunal estuvo regido de acuerdo a la zona y a la legislación vigente, aplicando Mareck, Newcastle y Gumboro el día cero (0) de vida (en incubadora); y refuerzo de Newcastle y Gumboro, los días 12 y 24 de vida (Newcastle: vía ocular; Gumboro: oral).

\section{DiETAS}

Los animales fueron alimentados con cinco dietas: dieta comercial con y sin la adición de antibiótico, esta última con adición de tres diferentes concentraciones de AEO (L.origanoides) así:

Dieta 1 (Control): Alimento comercial sin antibiótico (AC), sin adición AEO.

Dieta 2: Alimento comercial con antibiótico, sin adición de AEO.

Dieta 3: AC + 75ppm de AEO.

Dieta 4: AC + 100 ppm de AEO. 
Dieta 5: AC + 200 ppm de AEO.

Se elaboró un alimento multietapa que cumplía con los requerimientos mínimos nutricionales establecidos por la guía de manejo COBB500 (2012)) (Tabla I). El alimento utilizado en el estudio estuvo libre de antibióticos (excepto la dieta D2, donde se utilizó Bacitracina de Zinc a razón de 400 gr por tonelada como APC, como se realiza a nivel comercial). Los animales tuvieron acceso a agua a voluntad durante todo el tiempo experimental. Los pollos consumieron las dietas que les correspondieron desde el día 1 del experimento.

\section{SACRIFICIO}

Durante la fase de experimentación se realizaron eutanasias escalonadas de la siguiente forma: los días 14,28 y 42 se sacrificaron cinco aves por tratamiento. Todas las aves fueron sacrificadas 2.5 horas después de su última comida. Los animales se sedaron por inhalación de Nitrox y posteriormente se les realizó eutanasia humanitaria con dióxido de carbono durante 3 minutos.

Después del sacrificio, las aves se colocaron en posición decúbito dorsal, boca arriba, y extendiendo las alas lateralmente; luego se realizó un corte desde la parte anterior del cuello hasta la cloaca, cortando solo la piel. Se realizaron dos pequeños cortes laterales hasta llegar a las costillas, y luego se hizo un corte de las costillas en dirección craneal. Se evaluó la presencia de exudados diversos y el estado de sacos aéreos. Posteriormente, se extrajo completamente el intestino delgado, desde la unión pilórica hasta la válvula íleo-cecal (Segalés \& Domingo, 2003). El intestino fue alineado y medido en una mesa sin ningún tipo de tensión; posteriormente éste se dividió en tres regiones (duodeno, yeyuno, e íleon) (Reis, Guerrero, Aguilera, \& Mariscal, 2005), y se tomaron $2 \mathrm{~cm}$ del centro de cada sección.Una vez cortadas estas secciones, se hizo un lavado por infusión con solución salina fría de cada porción removida para eliminar impurezas y la digesta contenida en órganos digestivos y fueron almacenadas en formalina al 10\% (Rodríguez-González \& MorenoFigueredo, 2016)

\section{TOMA DE MUESTRAS}

Análisis morfométrico del intestino delgado: Transcurrido 48 horas después de la colecta, las muestras de las diferentes secciones del intestino almacenadas en formalina al $10 \%$, fueron enviadas al laboratorio de Histopatología Animal de la Universidad de Antioquia (Facultad de Ciencias Agrarias) para ser analizadas por expertos.

Procesamiento histotécnico: Los tejidos fueron fijados en formalina tamponada al $10 \%$ por 48 horas a $4^{\circ} \mathrm{C}$, incluidos en parafina, cortados a $4 \mu \mathrm{m}$ de espesor $\mathrm{y}$ coloreados con Hematoxilina-Eosina para ser lavados y almacenados en etanol:agua (75:25, v:v) de acuerdo con el método reportado por Vente-Spreeuwenberg et al. (2003). Estos cortes fueron microdiseccionados para determinar el promedio de la altura y ancho de las vellosidades intestinales, así como la profundidad y ancho de las criptas adyacentes. En cada lámina se montaron tres cortes transversales.
Tabla I. Composición nutricional de la dieta basal (Nutritional composition of the basal diet).

\begin{tabular}{|c|c|c|}
\hline Materia prima & Cantidad & $\%$ \\
\hline Lisina HCL & 2,4 & 0,238 \\
\hline Metionina DL & 2,5 & 0,246 \\
\hline Bentonina DL & 4 & 0,4 \\
\hline Cloruro de colina $60 \%$ & 0,5 & 0,05 \\
\hline Sal yodada & 2 & 0,2 \\
\hline Treonina- L & 0,7 & 0,066 \\
\hline Fosfato monodicálcico 21\% & 6,6 & 0,656 \\
\hline Harina de carne $50 \mathrm{P} / 17 \mathrm{G} / 18 \mathrm{C}$ & 15 & 1,5 \\
\hline Carbonato de calcio & 15 & 1,5 \\
\hline Harina de arroz 8-18 & 26,6 & 2,664 \\
\hline Soya 18 integral & 88,3 & 8,83 \\
\hline Torta de soya $48 \%$ & 250 & 25 \\
\hline Maiz S-12 & 586,5 & 58,65 \\
\hline Total & 1000 & 100 \\
\hline Nutrientes (\%) & \multicolumn{2}{|c|}{ Valor } \\
\hline Peso (Kg) & \multicolumn{2}{|c|}{1} \\
\hline Humedad & \multicolumn{2}{|c|}{11,071} \\
\hline $\begin{array}{l}\text { Energía metabolizable Aves } \\
\mathrm{KCAL} / \mathrm{Kg}\end{array}$ & \multicolumn{2}{|c|}{3005.940} \\
\hline Proteina bruta & \multicolumn{2}{|c|}{19,582} \\
\hline Grasa & \multicolumn{2}{|c|}{5,045} \\
\hline Ácidos grasos saturados & \multicolumn{2}{|c|}{0,352} \\
\hline ácidos grasos insaturados & \multicolumn{2}{|c|}{2,123} \\
\hline Materia seca & \multicolumn{2}{|c|}{88,929} \\
\hline Extracto libre de Nitrógeno & \multicolumn{2}{|c|}{55,508} \\
\hline Fibra bruta & \multicolumn{2}{|c|}{2,769} \\
\hline Cenizas & \multicolumn{2}{|c|}{6,022} \\
\hline Calcio & \multicolumn{2}{|c|}{0,84} \\
\hline Fósforo disponible & \multicolumn{2}{|c|}{0,294} \\
\hline Fósforo total & \multicolumn{2}{|c|}{0,546} \\
\hline Cloro & \multicolumn{2}{|c|}{0,218} \\
\hline Sodio & \multicolumn{2}{|c|}{0,123} \\
\hline Potasio & \multicolumn{2}{|c|}{0,832} \\
\hline Balance Electrolítico (mEg/kg) & \multicolumn{2}{|c|}{204,709} \\
\hline
\end{tabular}

Evaluación microscópica y análisis morfométrico de imágenes:Los cortes histológicos fueron analizados cuantitativamente mediante un procesamiento de imágenes digitales computarizadas, así: para la identificación de las zonas tisulares se empleó un microscopio óptico Leica DLMB(Meyer, Houston, TX, USA), luego se capturaron las imágenes correspondientes con una cámara para microscopía digital instantánea Motican 2300 (Motic, Hong Kong, China) con una resolución de 3 megapixeles, en un aumento de 200x y se analizaron dichas imágenes con el software para tratamiento de imágenes Motic ${ }^{\circledR}$ Images plus 2.0 (Motic, Hong Kong, China). 


\begin{tabular}{lc}
\hline \multicolumn{2}{l}{ Tabla I (cont.). Composición nutricional de la dieta } \\
basal (Nutritional composition of the basal diet). \\
\hline Lisina & 1,301 \\
Metionina & 0,543 \\
Metionina + Cisteína & 0,871 \\
Treonina & 0,835 \\
Triptofano & 0,242 \\
Arginina & 1,376 \\
Isoleucina & 0,85 \\
Leucina & 1,707 \\
Valina & 0,956 \\
Histidina & 0,547 \\
Fenilalanina & 1 \\
Fenilalanina + Tirosina & 1,835 \\
Glicina & 0,982 \\
Alanina & 1,107 \\
Lisina digestible en aves & 1,177 \\
Metionina digestible en aves & 0,519 \\
Metionina + Cisteina digestible en aves & 0,724 \\
Treonina digestible en aves & 0,793 \\
Triptófano digestible en aves & 0,804 \\
Isoleucina digestible en aves & 0,365 \\
Leucina digestible en aves & 0,201 \\
Valina digestible en aves & 1,247 \\
\hline
\end{tabular}

Las variables morfológicas que se midieron en cada corte histológico de las vellosidades intestinales fueron:

1. Altura: una vez se estableció la base de la vellosidad, desde su punto medio se trazó una línea hasta el ápice.

2. Ancho: con una línea se unieron los bordes apicales de las células epiteliales de lados opuestos, ubicadas aproximadamente en la mitad de la vellosidad.

También se determinó la profundidad y el ancho de las criptas intestinales, conforme a lo descrito previamente por Marion et al. (2002) y Vente-Spreeuwenberg et al (2003).

\section{ANÁLISIS ESTADÍSTICO}

Se realizó según un diseño bloques al azar en un arreglo de parcelas divididas. Para la conformación de los bloques se tomó en consideración la ubicación geográfica en el galpón de los animales. A cada animal le fue asignado uno de los 15 tratamientos (5 dietas experimentales y tres periodos de evaluación). Cada tratamiento tuvo un total de 5 repeticiones (aves por corral). El análisis estadístico se realizó según el procedimiento GLM (Modelos Lineales Generales) del SAS (Statistical Analysis Systems Institute , 2007, ) (2007). Las diferencias entre las medias de los tratamientos fueron determinadas por mínimos cuadrados y analizadas por ANOVA (Raji, Adeleye, Mosobalaje, Ogunjimi, \& Tewe, 2016). Para realizar la comparación de los promedios entre tratamientos se utilizó una prueba de Duncan $(\mathrm{P}<0.05)$.

\section{RESULTADOS}

En general, las aves que consumieron los diferentes alimentos presentaron un buen estado de salud, no presentaron ningún síntoma o signo adverso de enfermedad que causara su retiro y/o sacrificio. Adicionalmente, las aves consumieron la ración diaria de alimento ajustada a la guía de manejo COBB500 (2012).

En esta investigación no se encontró interacción estadística entre las diferentes dietas y los días de sacrificio para ninguna de las variables en estudio, por ello no fue necesario desglosar y analizar cada uno de los factores de forma independiente.

En las Figuras 1, 2 y 3, se pueden observar los cambios morfométricos de las vellosidades de duodeno, entre las diferentes dietas (blanco, APC y los tres niveles de inclusión de AEO) en el día 42. Estos cambios, representados por los valores de longitud y el ancho de las vellosidades entre cada una de las dietas y los períodos de exposición, se muestran en la Tabla II. Respecto a la longitud y ancho de las vellosidades, se observó un incremento significativo $(\mathrm{P}<0,01)$ entre las diferentes dietas evaluadas, donde D1 reportó los valores más bajos, para ambos criterios evaluados y en todos los periodos de evaluación, en comparación con $\mathrm{D} 2$ y con las dietas con adición de AEO, donde los animales que consumieron D5 reportaron los mayores valores para estas variables intestinales.

Al comparar los diferentes segmentos intestinales se presentó diferencia estadísticamente significativa $(\mathrm{P}<0,01)$, donde el segmento duodeno mostró los valores más altos en comparación con los otros dos segmentos (yeyuno e íleon) respecto a longitud y ancho de las vellosidades. Para las mismas variables que fueron objeto de estudio, hubo diferencia estadísticamente significativa entre los diferentes días de muestreo en cada una de las dietas $(\mathrm{P}<0,05)$, donde se encontraron los valores más altos en el día 42.

Los resultados de la evaluación de profundidad y ancho de criptas se presentan en la Tabla III. En cuanto a la profundidad de criptas hubo diferencia estadística significativa $(\mathrm{P}<0.05)$ entre todas las edades y todos los tratamientos para los tres segmentos intestinales analizados, siendo los resultados de menor profundidad de criptas encontrados con la mayor inclusión de AEO. Para el ancho de las criptas, donde valores menores indican mejor salud del epitelio intestinal, se reportaron diferencias significativas en todas las edades y todas 


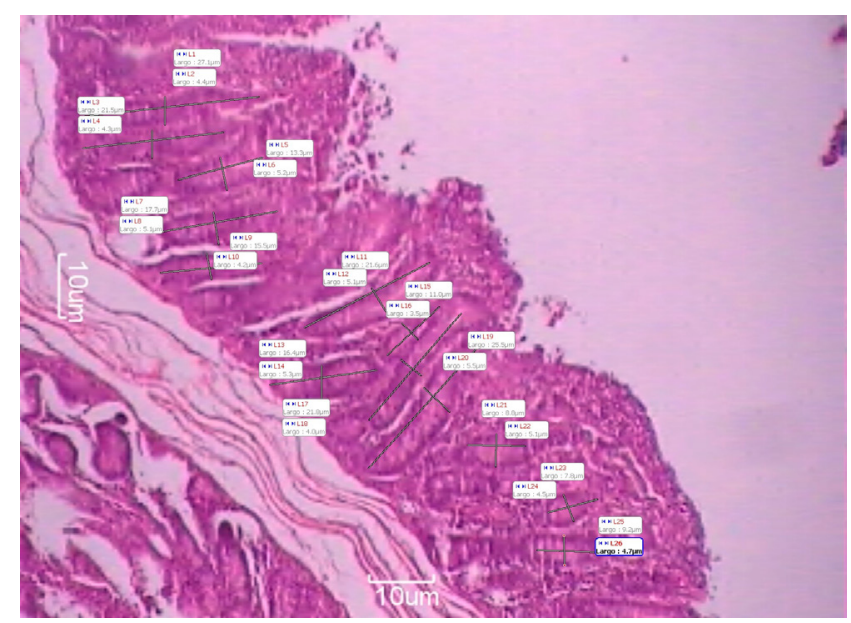

Figura 1. Vellosidades Dieta control (Control diet Villi).

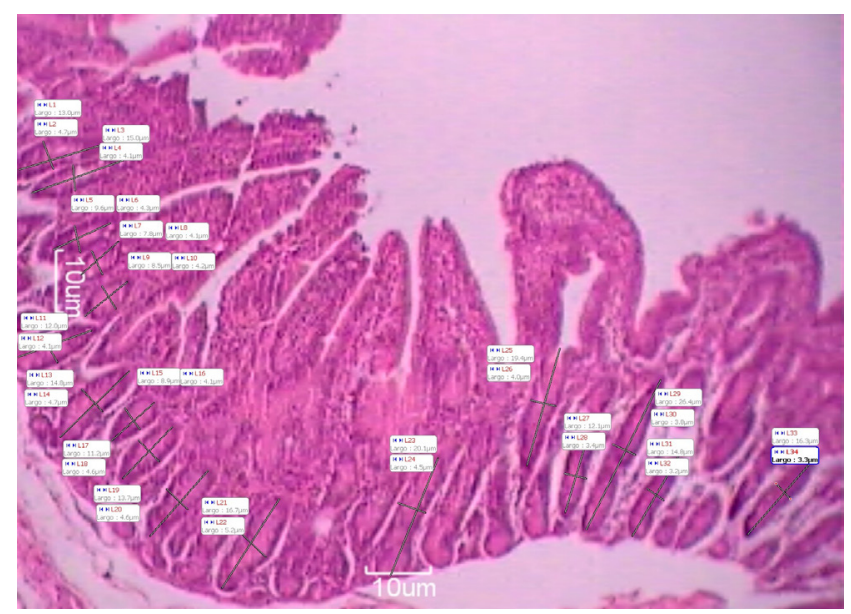

Figura 3. Vellosidades Dieta 5 (Diet 5 Villi).

las dietas de los tres segmentos del intestino, excepto para D2 y D3 en día 42 del duodeno.

En general para profundidad y ancho de criptas los resultados de D2 fueron mejores a la dieta control (D1), pero las dietas con AEO (Lippia origanoides) tuvieron los mejores resultados, destacándose la dieta D5.

\section{DISCUSIÓN}

En esta investigación se encontró un efecto positivo de la adición de AEO de Lippia origanoides tipo timol sobre la morfología intestinal de pollos machos de engorde de línea Cobb500, ya que aumenta el tamaño en largo y ancho de las vellosidades y disminuye en tamaño (profundidad y ancho) las criptas, logrando que el pollo tenga un intestino con más capacidad de absorción, mayor área de cobertura y mejores condiciones para flora benéfica, además de menor demanda de energía para renovación del tejido epitelial. Con respecto a vellosidades, García et al. (2007) encontraron efectos positivos sobre la altura de la vellosidad y la profundidad de la cripta en pollos suplementados con 200 ppm de una mezcla de orégano, canela y pimienta; agentes antimicrobianosreconocidos por reducir la carga microbiana, lo cual a la vez disminuye la presencia de toxinas que son asociadas con cambios en la morfología intestinal, como el acortamiento de la

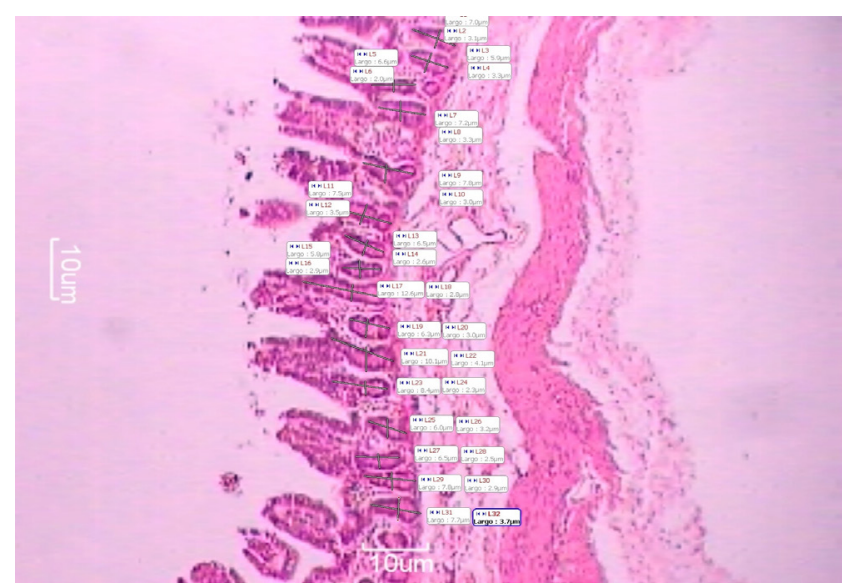

Figura 2. Vellosidades Dieta 2 (Diet 2 Villi).

vellosidad y aumento en la profundidad de la cripta. Una cripta profunda indica un rápido intercambio de tejido y una demanda alta para la renovación del tejido epitelial, por lo tanto, se incrementan los requerimientos nutricionales y se disminuye la eficiencia productiva del animal (Roldan, 2010).

Una vellosidad corta disminuye la superficie de absorción de nutrientes; un alargamiento de la vellosidad indica una rápida reconversión del tejido y una alta demanda por nuevos tejidos (Jaramillo A. , 2014). Por otra parte, los factores involucrados en la integridad del intestino, tienen consecuencias importantes para la eficiencia alimenticia, debido a que la capacidad de la absorción de nutrientes de cada segmento del intestino, es proporcional al número, altura y ancho de las vellosidades presentes, ya que definen el tamaño y área de la superficie disponible para la absorción de nutrientes (Pelicano, y otros, 2003) (Rodríguez-González \& Moreno-Figueredo, 2016).Los resultados obtenidos ,se asemejan a los reportados por Jaramillo (2011), quién evaluó el efecto de adicionar un ácido orgánico, un prebiótico y su uso sinérgico comparado con una dieta que contenía APC (Bacitracina de Zinc) y una dieta blanco en un período de 22 días con muestreos los días 8,15 y 22. Los valores encontrados por Jaramillo (2011) se asemejan a los encontrados en esta investigación cuando se incluyó AEO, mostrando que los prebióticos, los ácidos orgánicos y el AEO tienen un efecto directo sobre la morfología intestinal de pollos de engorde.

\section{CONCLUSIÓN}

El AEO de Lippia origanoides mostró tener un efecto positivo sobre las vellosidades (más largas) y las criptas (menos profundas) de pollos de engorde de la línea genética Cobb500 si compara con una dieta basal y con una dieta adicionada con antibiótico promotor de crecimiento.

El AEO de Lippia origanoides tipo timol se constituye como una alternativa viable para el reemplazo de los APC, siendo un promotor nutricional de crecimiento de origen vegetal que mejorapositivamente la morfología de las vellosidades (más largas y anchas) y criptas (menos profundas y anchas) de los tres segmentos del 


\begin{tabular}{|c|c|c|c|c|c|c|c|c|}
\hline Vellosidad $(\mu \mathrm{m})$ & Segmento intestinal & Edad & D1 & $\mathrm{D} 2$ & D3 & D4 & D5 & EEM \\
\hline \multirow{8}{*}{$\frac{\stackrel{\pi}{\stackrel{3}{2}}}{\stackrel{+}{<}}$} & \multirow{3}{*}{ Duodeno $^{1}$} & 14 & $635,7^{A, X}$ & $698,6^{\mathrm{B}, \mathrm{X}}$ & $741,5^{c, x}$ & $784,7^{\mathrm{D}, \mathrm{x}}$ & $812,3 \mathrm{E}, \mathrm{X}$ & \multirow{9}{*}{3.34} \\
\hline & & 28 & $681,2^{A, Y}$ & $738,2^{B, Y}$ & $767,3^{\mathrm{C}, \mathrm{Y}}$ & $809,1^{D, Y}$ & $851,9 \mathrm{E}, \mathrm{Y}$ & \\
\hline & & 42 & $746,7^{A, Z}$ & $797,8^{\mathrm{B}, \mathrm{Z}}$ & $815,1^{c, z}$ & $839,5^{\mathrm{D}, \mathrm{Z}}$ & $879,5 \mathrm{E}, \mathrm{Z}$ & \\
\hline & \multirow{3}{*}{ Yeyuno $^{2}$} & 14 & $627,5^{\mathrm{A}, \mathrm{X}}$ & $672,6^{\mathrm{B}, \mathrm{X}}$ & $701,5^{c, x}$ & $752,1^{D, x}$ & $778,5^{\mathrm{E}, \mathrm{X}}$ & \\
\hline & & 28 & $653,4^{A, Y}$ & $699,7^{B, Y}$ & $747,7^{\mathrm{C}, \mathrm{Y}}$ & $784,4^{\mathrm{D}, \mathrm{Y}}$ & $806,5^{E, Y}$ & \\
\hline & & 42 & $689,3^{A, Z}$ & $720,8^{\mathrm{B}, \mathrm{Z}}$ & 773,9 c,z & $826,7^{\mathrm{D}, \mathrm{Z}}$ & $831,5^{\mathrm{D}, \mathrm{Z}}$ & \\
\hline & \multirow{3}{*}{ Ileon $^{3}$} & 14 & $607,5^{\mathrm{A}, \mathrm{X}}$ & $643,7^{\mathrm{B}, \mathrm{X}}$ & $661,9^{\mathrm{B}, \mathrm{X}}$ & $714,7^{c, x}$ & $753,2^{D, X}$ & \\
\hline & & 28 & $619,9^{A, X}$ & $664,3^{B, X Y}$ & $696,2^{\mathrm{C}, \mathrm{Y}}$ & $739,8^{D, Y}$ & $781,5^{\mathrm{D}, \mathrm{Y}}$ & \\
\hline \multirow{10}{*}{$\begin{array}{l}\frac{0}{0} \\
\frac{1}{0}\end{array}$} & & 42 & $636,3^{A, Y}$ & 674,9 B,Y & $725,5^{c, z}$ & $774,9^{\mathrm{D}, \mathrm{Z}}$ & $801,8^{E, Z}$ & \\
\hline & \multirow{3}{*}{ Duodeno $^{1}$} & 14 & $131,5^{\mathrm{A}, \mathrm{X}}$ & $142,4^{B, X}$ & $157,1^{c, x}$ & $165,7^{\mathrm{D}, \mathrm{X}}$ & $183,1 \mathrm{E}, \mathrm{X}$ & \multirow{9}{*}{1.23} \\
\hline & & 28 & $141,2^{A, Y}$ & $156,5^{\mathrm{B}, \mathrm{XY}}$ & $170,6^{c, Y}$ & $182,6^{\mathrm{D}, \mathrm{Y}}$ & $199,3 \mathrm{E}, \mathrm{Y}$ & \\
\hline & & 42 & $154,9^{A, Z}$ & $166,6^{B, Y}$ & $186,1^{c, z}$ & $207,5^{\mathrm{D}, \mathrm{Z}}$ & $211,5^{\mathrm{D}, \mathrm{Z}}$ & \\
\hline & \multirow{3}{*}{ Yeyuno $^{2}$} & 14 & $125,1^{A, X}$ & $134,2^{B, X}$ & $142,7^{\mathrm{B}, \mathrm{X}}$ & $147,5^{c, x}$ & $163,1^{D, X}$ & \\
\hline & & 28 & $129,7^{A, X Y}$ & $145,8^{B, Y}$ & $152,7^{B, Y}$ & $167,6^{\mathrm{C}, \mathrm{Y}}$ & $179,4^{D, Y}$ & \\
\hline & & 42 & $135,3^{A, Y}$ & $159,4^{\mathrm{B}, \mathrm{Z}}$ & $161,7^{\mathrm{B}, \mathrm{Z}}$ & $180,7^{c, z}$ & $191,7^{\mathrm{D}, \mathrm{z}}$ & \\
\hline & \multirow{3}{*}{ lleon $^{2}$} & 14 & $115,2^{A, X}$ & $128,4^{B, X}$ & $136,71^{c, x}$ & $140,2^{\mathrm{D}, \mathrm{x}}$ & 157,3 E,X & \\
\hline & & 28 & $123,3^{A, X Y}$ & $134,9^{B, X}$ & $149,8^{c, Y}$ & $164,2^{D, Y}$ & 179,4 E,Y & \\
\hline & & 42 & $133,4^{A, Y}$ & $143,4^{\mathrm{B}, \mathrm{Y}}$ & $158,89 \mathrm{c}, \mathrm{z}$ & $182,2^{\mathrm{D}, \mathrm{Z}}$ & 195,4 E,Z & \\
\hline
\end{tabular}

Dieta 1: Alimento comercial sin antibiótico (AC), sin adición AEO. Dieta 2: Alimento comercial con antibiótico, sin adición de AEO. Dieta 3: AC + 75ppm de AEO. Dieta 4: AC + 100 ppm de AEO. Dieta 5: AC + 200 ppm de AEO. A,B,C,D, E Dentro de una misma fila, medias con diferente letra son estadísticamente diferentes $(P<0.05)$. ${ }^{X, Y, Z}$ Dentro de una misma columna, medias con diferente letra son estadísticamente diferentes $(P<0.05){ }^{1,2,3}$ Dentro de una misma columna con un superíndice diferente (por segmento intestinal en estudio) difieren estadísticamente $(P<0.05)$. EEM: Error estándar de la media.

Tabla III. Morfometría en criptas intestinales de pollos con y sin adición de AEO (Morphometry in intestinal crypts of chickens with and without the addition of AEO).

\begin{tabular}{|c|c|c|c|c|c|c|c|c|}
\hline Criptas $(\mu \mathrm{m})$ & Segmento intestinal & Edad & D1 & D2 & D3 & D4 & D5 & EEM \\
\hline \multirow{9}{*}{$\begin{array}{l}\frac{0}{\pi} \\
\frac{\pi}{0} \\
\frac{0}{0} \\
\frac{0}{2} \\
\frac{0}{0} \\
\end{array}$} & \multirow{3}{*}{ Duodeno $^{1}$} & 14 & $147,2^{A, X}$ & $142^{\mathrm{B}, \mathrm{X}}$ & $140,4^{c, x}$ & $134,1^{D, X}$ & $130,4 \mathrm{E}, \mathrm{X}$ & \multirow{9}{*}{1.04} \\
\hline & & 28 & $143,2^{A, Y}$ & $134,2^{B, Y}$ & $129,5^{c, Y}$ & $120,2^{D, Y}$ & $119,5 \mathrm{E}, \mathrm{Y}$ & \\
\hline & & 42 & $138,2^{A, Z}$ & $124,4^{\mathrm{B}, \mathrm{Z}}$ & $115,6^{c, z}$ & $107,3^{\mathrm{D}, \mathrm{z}}$ & $100,6 \mathrm{E}, \mathrm{Z}$ & \\
\hline & \multirow{3}{*}{ Yeyuno $^{2}$} & 14 & $168^{A, X}$ & $163,6^{\mathrm{B}, \mathrm{X}}$ & $154,1^{c, x}$ & $146,5^{\mathrm{D}, \mathrm{X}}$ & $133,3 \mathrm{E}, \mathrm{X}$ & \\
\hline & & 28 & $158,5^{\mathrm{A}, \mathrm{Y}}$ & $153,5^{B, Y}$ & $144,5^{\mathrm{C}, \mathrm{Y}}$ & $137,8^{D, Y}$ & $128,3^{E, Y}$ & \\
\hline & & 42 & $151^{\mathrm{A}, \mathrm{Z}}$ & $145,4^{\mathrm{B}, \mathrm{Z}}$ & $136,9^{c, z}$ & $130,1^{\mathrm{D}, \mathrm{Z}}$ & $124,3 \mathrm{E}, \mathrm{Z}$ & \\
\hline & \multirow{3}{*}{ lleon $^{3}$} & 14 & $174,4^{\mathrm{A}, \mathrm{X}}$ & $167,1^{\mathrm{B}, \mathrm{X}}$ & $157,3^{c, x}$ & $152,3^{D, X}$ & $145,5^{\mathrm{E}, \mathrm{X}}$ & \\
\hline & & 28 & $166,2^{\mathrm{A}, \mathrm{Y}}$ & $156,7^{\mathrm{B}, \mathrm{Y}}$ & $149,5^{c, Y}$ & $143,1^{\mathrm{D}, \mathrm{Y}}$ & $137,4 \mathrm{E}, \mathrm{Y}$ & \\
\hline & & 42 & $158^{\mathrm{A}, \mathrm{Z}}$ & $149,3^{\mathrm{B}, \mathrm{Z}}$ & $141,7^{c, z}$ & $135,9^{\mathrm{D}, \mathrm{Z}}$ & $129,3 \mathrm{E}, Z$ & \\
\hline \multirow{9}{*}{$\begin{array}{l}\frac{O}{0} \\
\frac{\mathrm{C}}{<}\end{array}$} & \multirow{3}{*}{ Duodeno $^{1}$} & 14 & $141,1^{A, x}$ & $135,3^{B, x}$ & $132,4^{c, x}$ & $127,6^{\mathrm{D}, \mathrm{X}}$ & $124,8 \mathrm{E}, \mathrm{X}$ & \multirow{9}{*}{0.58} \\
\hline & & 28 & $134,6^{\mathrm{A}, \mathrm{Y}}$ & $128,1^{B, Y}$ & $123,8^{c, Y}$ & $119,6^{\mathrm{D}, \mathrm{Y}}$ & $112,5^{E, Y}$ & \\
\hline & & 42 & $126,1^{\mathrm{A}, \mathrm{Z}}$ & $118,9^{B, Z}$ & $117,2^{\mathrm{B}, \mathrm{Z}}$ & $111,6^{c, z}$ & $104,1^{\mathrm{D}, \mathrm{Z}}$ & \\
\hline & \multirow{3}{*}{ Yeyuno $^{2}$} & 14 & $147,7^{\mathrm{A}, \mathrm{X}}$ & $141,5^{\mathrm{B}, \mathrm{X}}$ & $137,2^{c, x}$ & $132,5^{\mathrm{D}, \mathrm{X}}$ & $129,9 \mathrm{E}$ & \\
\hline & & 28 & $137,2^{A, Y}$ & $132,5^{B, Y}$ & $127,5^{c, Y}$ & $123,1^{D, Y}$ & $119,3 \mathrm{E}$ & \\
\hline & & 42 & $128,7^{A, Z}$ & $127,5^{\mathrm{B}, \mathrm{Z}}$ & $121,8^{c, z}$ & $119,7^{\mathrm{D}, \mathrm{Z}}$ & $112,7^{\mathrm{E}}$ & \\
\hline & \multirow{3}{*}{ lleon $^{3}$} & 14 & $148,2^{\mathrm{A}, \mathrm{X}}$ & $144,8^{\mathrm{B}, \mathrm{X}}$ & $141,2^{c, x}$ & $136,2^{\mathrm{D}, \mathrm{X}}$ & $133,6 \mathrm{E}, \mathrm{X}$ & \\
\hline & & 28 & $141,3^{A, Y}$ & $139,2^{B, Y}$ & $134,5^{c, Y}$ & $129,7^{\mathrm{D}, \mathrm{Y}}$ & $124,3 \mathrm{E}, \mathrm{Y}$ & \\
\hline & & 42 & $136,4^{A, Z}$ & $132,2^{\mathrm{B}, \mathrm{Z}}$ & $130,2^{c, z}$ & $124,2^{\mathrm{D}, \mathrm{Z}}$ & 117,3 E,Z & \\
\hline
\end{tabular}

Dieta 1: Alimento comercial sin antibiótico (AC), sin adición AEO. Dieta 2: Alimento comercial con antibiótico, sin adición de AEO. Dieta 3: $A C+75 p p m$ de AEO. Dieta 4: AC + 100 ppm de AEO. Dieta 5: AC + 200 ppm de AEO. A,B,C,D,E Dentro de una misma fila, medias con diferente letra son estadísticamente diferentes $(P<0.05)$. ${ }^{X, Y, Z}$ Dentro de una misma columna, medias con diferente letra son estadísticamente diferentes $(P<0.05)$. 1,2,3 Dentro de una misma columna con un superíndice diferente (por segmento intestinal en estudio) difieren estadísticamente $(P<0.05)$. EEM: Error estándar de la media. 
intestino delgado (duodeno, yeyuno e íleon) durante el ciclo de producción de carne con pollos de engorde.

\section{BIBLIOGRAFÍA}

Betancourt $L$ 2012. Evaluación de aceites esenciales de orégano en la dieta de pollos de engorde", [Tesis de Doctorado en Ciencias]. Bogotá, Colombia.: Universidad Nacional de Colombia, Facultad de Medicina Veterinaria y Zootecnia.

Cervantes M 201 1. Integridad intestinal en aves". Phibro Animal Health Corporation, Watkinsville, Ga., EUA. Industria Avícola.

CIOMS 2012. COUNCIL FOR INTERNATIONAL ORGANIZATIONS OF MEDICAL SCIENCES (CIOMS). International Guiding Principles for Biomedical Research Involving Animals. Geneva (Italy).

COBB VANTRESS . (2012). COBB Guía de Manejo del Pollo de Engorde. COBB VANTRESS .

García V, Catalá-Gregori P, Hernández F, Megías M, \& Madrid J 2007. "Effect of formic acid and plant extracts on growth, nutrient digestibility, intestine mucosa morphology and meat yield of broiler", . The Journal of Applied Poultry Research, vol 16, no. 4 Abril, pp. 555- 562.

García-Curbelo Y, \& García-Hernández Y 2015. Uso de aditivos en la alimentación animal: 50 años de experiencia en el Instituto de Ciencia Animal. Revista Cubana de Ciencia Agrícola, Tomo 49, Número 2, pp. 173-177.

Jaramillo A 2014. Evaluación de un prebiótico y ácido orgánico, en el crecimiento alométrico del sistema digestivo, morfometría de vellosidades y bacterias intestinales en pollos de engorde. Plumazos, Amevea. no. 50, Diciembre, pp. 4-24., pp. 4-24.

Jaramillo, B 2011 . Evaluacion de la mezcla de un prebiotico y un ácido orgánico en la salud intestinal y parámetros productivos de pollos de engorde. [Tesis de Maestría en Ciencias Agrarias]. Ibagué, Tolima: Universidad Nacional de Colombia sede Palmira.

Maguiña-Vargas, C 2013. Uso racional de antibióticos. Lima-Perú: Depósito Legal en la Biblioteca Nacional del Perú: 2012-09186.

Marion J, Biernat M, Thomas F, Savary G, Le Breton Y, Zabielskil R, Le Huërou-Luron I \& Le Dividich, J 2002. Small intestine growth and morphometry in piglets weaned at 7 days of age. Effects of level of energy intake. Reproduction Nutrition Development, vol 42, no. 4, julio-agosto, , pp. 339-354. .

Martínez I, Ruiz N, \& Zambrano R 2008. "Efecto inmunoestimulante de mix-oil en pollos de engorde". Semillero de ciencias veterinarias aplicadas (INAMEVEZ).

Ojediran T, Fasola M, Oladele T, Onipede T, \& Emiola I 2017. Growth performance, flock uniformity and economic indices of broiler chickens fed low crude protein diets supplemented with lysine. Archivos de Zootecnia. 66 (256), 543-550.

Oloruntola O, Ayodele S, Agbede J, \& Oloruntola D 2016 . Effect of feeding broiler chickens with diets containing Alchornea cordifolia leaf meal and enzyme supplementation. Archivos de Zootecnia. 65 (252), 489-498.
Pelicano E, Alves PA., Oba A, Norkus E, Kodawara L \& Azevedo T 2003. Morfometria e ultra-estrutura da mucosa intestinal de frangos de corte alimentados com dietas contenido diferentes prebióticos. Revista Portuguesa de Ciências Veterinárias, vol 98, no. 547, pp. 125-34. .

Raji M, Adeleye O, Mosobalaje M, Ogunjimi J \& Tewe O 2016. Growth response and serum biochemical parameters of starter broiler chickens fed toasted African yam bean (Sphenostylis stenocarpa) seeds meal with enzyme supplementation. Archivos de Zootecnia, 65 (250): , 139-143.

Reis S, Guerrero C, Aguilera B \& Mariscal L 2005. Efecto de diferentes cereales sobre la morfología intestinal de lechones recién destetados. Técnica Pecuaria en México, vol 43, no. 3, septiembre - diciembre, pp. 309-321.

Rodríguez-González SP \& Moreno-Figueredo G 2016. Evaluación del efecto de Lactobacillus spp. en el desarrollo del intestino delgado en pollos de engorde. Revista Ciencia y Agricultura Vol. 13 (1). ISSN 0122-8420. , pp. 49-58.

Roldan L 2010. Evaluación del uso de los aceites esenciales como alternativa al uso de los antibióticos promotores de crecimiento en pollos de engorde. [Tesis de Maestría en Producción Animal] Bogotá: Universidad Nacional de Colombia.

Segalés, J \& Domingo M 2003. La necropsia en el ganado porcino, diagnóstico anatomopatológico y toma de muestras. Madrid, España, pp. 10-14.: Boehringer Ingelheim.

Sergeant M., Constantinidou C, Cogan T, Bedford M, Penn C \& Pallen M 2014. Extensive Microbial and Functional Diversity within the Chicken Cecal Microbiome. Plos One. DOI: 10.1371/journal.pone.0091941. Skoufos I, Giannenas I, Tontis D, Bartzanas T, Kittas C, Panagakis P \& Tzora A 2016. Effects of oregano essential oil and attapulgite on growth performance, intestinal microbiota and morphometry in broilers. South African Journal of Animal Science 46 (No. 1), 77-89.

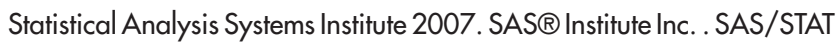
User's Guide, Version 9.1 th Ed. Cary, NC: SAS Institute Inc.

Tavernari F, Salguero S, Albino L \& Rostagno H 2008. "Nutrición, patología y fisiología digestiva en pollos: aspectos prácticos", Nutrición, patología y fisiología digestiva en pollos: aspectos prácticos XXIV, (pág. pp. 16.). Madrid: Curso de especialización FEDNA.

Vente-Spreeuwenberg M, Verdonk J, Verstegen M \& Beynen A 2003. Villus height and gut development in weaned piglets receiving diets containing either glucose, lactose or starch". British Journal of Nutrition vol 90, no. 5, noviembre, pp. 907-9. 